\title{
Relación entre el sexo y el estrato sociodemográfico de los habitantes de los distritos de San Isidro y Cercado de Lima con la percepción de la inseguridad ciudadana
}

Relationship between sex and the sociodemographic stratum of the inhabitants of the districts of San Isidro and Cercado de Lima with the perception of citizen insecurity

\author{
Alison Aguilar Inga ${ }^{1}$, Cristina Bueno Araujo ${ }^{1}$, Eder Bautista Bravo ${ }^{1}$, Emily Cárdenas Hiraoka ${ }^{1}$, Tania \\ Chinguel Ordinola ${ }^{1}$, Víctor Hugo Ucedo Silva ${ }^{1}$.
}

\section{RESUMEN}

En este artículo se analizó la relación entre el sexo y estrato sociodemográfico de los habitantes que residen en los distritos de San Isidro y Cercado de Lima con la percepción de la inseguridad ciudadana. El estudio se basó en los datos obtenidos a partir de la encuesta realizada por el Instituto Nacional de Estadísticañ e Informática [INEI] sobre victimización en el período 2016-2017. La muestra estuvo conformada por 379 residentes habituales del distrito Cercado de Lima y 205 residentes habituales del distrito San Isidro. Los entrevistados tenían una edad de 15 años a más y fueron seleccionados de manera aleatoria. Los resultados indican que las variables de estrato sociodemográfico y distrito están relacionadas con varios indicadores de la percepción de inseguridad ciudadana, a diferencia de la variable sexo que sólo se relacionó con la variable de percepción referida a la existencia de vigilancia en el distrito por parte de la Policía Nacional.

PALABRAS CLAVE: Percepción de inseguridad, distrito, estrato sociodemográfico, Cercado de Lima, San Isidro.

\section{SUMMARY}

This article seeks to establish the correlation between sex and sociodemographic stratum of the residents of the districts of San Isidro and Cercado de Lima with the perception of citizen insecurity. A non-experimental design of descriptive-correlational type was used. The study was based on the data obtained from the survey conducted by the INEI on Victimization in 2016-2017. The sample consisted of 379 habitual residents of the Cercado de Lima district and 205 regular residents of the San Isidro district, and the persons were randomly selected from an age equal to or greater than 15 years. The results indicate that the socio-demographic and district stratum are related to most categories of the perception variable of citizen insecurity, unlike the variable sex, which only relates to the variable of perception of the existence of surveillance in their district by the National Police.

KEY WORDS: Perception of insecurity, district, socio-demographic stratum, Cercado de Lima, San Isidro.

\footnotetext{
Facultad de Psicologia, Universidad Peruana Cayetano Heredia. Lima, Perú.
} 


\section{INTRODUCCIÓN}

Kessler (2009) define la percepción de inseguridad como "la perturbación angustiosa del ánimo que se deriva de la diferencia entre el riesgo percibido de ser víctima de un crimen y la victimización de hecho" (p. 35). Existen una serie de conceptos relacionados con los cuales es importante establecer algunas diferencias. Ortiz de Urbina, Pareja, Ponce y Sibina (2006) plantearon una importante distinción entre la "preocupación por el delito" (percepción por la ciudadanía de la magnitud de la actividad delictiva) y "miedo a la victimización" (el miedo individual a ser víctima de un delito). En el presente estudio se presentarán datos que podrían clasificarse dentro de algunas de estas categorías y que agruparemos bajo el rótulo general de percepción de inseguridad.

Como observa Jasso (2013), la percepción de inseguridad no existe solamente en quienes ya han sido víctimas de un delito. Jasso también comenta que la inseguridad ciudadana tiene consecuencia una disminución de la vida social de las personas, quienes prefieren confinarse en sus hogares, lo que, a su vez, disminuye las oportunidades de vigilancia informal de las calles, generándose el círculo vicioso que favorece a la delincuencia. Otras tipo de consecuencias son económicas, ya que quienes se sienten posibles víctimas del delito deben invertir en medidas de seguridad.

Actualmente, se acepta que la percepción de inseguridad ha dejado de ser una característica solamente de ciertos ambientes urbanos para convertirse en un aspecto de gran prevalencia en las sociedades urbanas contemporánea (Garland, 2005), si bien, también se ha señalado que por su propia naturaleza, las ciudades son especialmente propensas a que se registren delitos en ellas (Ortiz et al., 2006).

Las encuestas de victimización son un instrumento usado internacionalmente para medir el grado de percepción de inseguridad. Jasso (2013), cita datos obtenidos por el Instituto Nacional de Estadística y Geografía de México, comprobando que en la mayoría de estados de esa república, la inseguridad ciudadana se percibe como el principal motivo de preocupación de la población y que más de 6 de cada 10 habitantes de ese país se sienten inseguros. En otros casos, el tópico de la seguridad se inserta en encuestas que analizan temas mucho más variados. Así, por ejemplo, una encuesta en la ciudad de Cali (Colombia), reveló que el $38 \%$ de sus habitantes se sienten inseguros en la ciudad. También se han realizado algunos estudios cualitativos acerca de esta problemática, como el de García-Castro y Villalobos (2016), en Costa Rica, el cual también reportó una "gran desconfianza en la seguridad pública".

En general, en América Latina, el miedo al delito es relativamente alto (Dammert, 2012). El Perú no es una excepción a esa realidad. Según el estudio de percepción realizado por la Encuesta de Lima cómo vamos (2018) sólo el 11,6\% de los habitantes de la ciudad de Lima, capital del Perú, se sienten seguros en la ciudad y $15,6 \%$ en su respectivo barrio. A nivel nacional, según el INEI (2017a), el 89\% de la población de 15 años a más siente que será víctima de un delito en los próximos 12 meses. Nuestro objetivo en el presente trabajo es determinar si la percepción de inseguridad varía en función del sexo y el estrato socioeconómico del habitante de la ciudad. Como se ha visto ya desde el pasado, los factores sociodemográficos juegan un papel importante en la percepción de riesgo (Hale, 1996). Nos proponemos investigar la incidencia del sexo, lugar de residencia y nivel socioeconómico en la percepción de los ciudadanos respecto de algunos indicadores de inseguridad.

\section{Métodología}

\section{Participantes}

Los datos se extrajeron de la Encuesta Nacional Especializada sobre Victimización llevada a cabo por el INEI durante el período 2016-2017 a nivel nacional. La muestra de estudio estuvo conformada por 584 participantes entre 15 años a más del área urbana en Lima Metropolitana de los cuales fueron considerados 379 habitantes del distrito del Cercado de Lima y 205 habitantes del distrito de San Isidro. La muestra fue seleccionada a través del método aleatorio de Kish. El estudio fue llevado a cabo durante el año 2017 (véase INEI, 2017b, para la ficha técnica de dicha encuesta). Es de notar que el Cercado de Lima alberga una población mayoritariamente de los estratos sociodemográficos medio o bajo, en tanto que San Isidro es básicamente un distrito residencial y comercial con una población ubicada entre los niveles sociodemográficos alto y medio. Ambos distritos cuentan con planes locales de seguridad ciudadana con estadísticas de incidencias de delitos e información acerca de lugares de riesgo en sus respectivas jurisdicciones (Comité Distrital de Seguridad Ciudadana de San Isidro, 2018; Municipalidad Metropolitana de Lima, 2017). 


\section{RESULTADOS}

\section{Estrato sociodemográfico}

Percepción de cuán seguros(as) se sienten en casa, según estrato sociodemográfico

Los habitantes del estrato sociodemográfico alto se sienten seguros en su casa en un $80,3 \%$, mientras que sólo el $0,96 \%$ hacen referencia a que se sienten muy inseguros. Asimismo, los habitantes del estrato sociodemográfico medio alto, mencionan que se sienten seguros en un 58,1\% y ninguno expresó sentirse muy inseguro. En el estrato sociodemográfico medio, el 53,6\% se sienten seguros en su casa y $42,4 \%$ de este grupo se sienten inseguros en su casa. En el caso de los habitantes del nivel sociodemográfico medio bajo, mencionan que se sienten seguros en un $54,2 \%$ y un $38,5 \%$, que se sienten inseguros en su casa. Los habitantes de estrato sociodemográfico medio bajo son quienes en un mayor porcentaje manifiestan que se sienten muy inseguros en su casa $(6,25 \%)$ en comparación con los demás estratos. Según estos resultados, la percepción de seguridad dentro del hogar se mantiene en más de la mitad de los encuestados en todos los estratos, pero es más alta en los estratos más altos.

Percepción respecto a cómo se sienten caminando de noche y solo(a) por su barrio, según estrato sociodemográfico

El $1,4 \%$ de los habitantes del estrato sociodemográfico alto se sienten muy seguros caminando de noche y solo por su barrio, 55,77\% se sienten seguros, $37,5 \%$ se sienten inseguros y $5,3 \%$ se sienten muy inseguros. Entre las personas del nivel sociodemográfico medio alto, el $0,78 \%$ se sienten muy seguros, el $17,8 \%$ se sienten seguros, se sienten inseguros el $67,4 \%$ y el $14,0 \%$ se sienten muy inseguros. En el estrato sociodemográfico medio, el $8.6 \%$ se sienten seguros, se sienten inseguros el $69,5 \%$ y el $21,9 \%$ se siente muy inseguro. Finalmente, en el estrato sociodemográfico medio bajo se sienten seguros el 9,4\%, inseguros caminando de noche y solo por su barrio se sienten el 70,8\% y $19,8 \%$ se sienten muy inseguros. En suma, sólo en el estrato alto la mayoría (algo más de la mitad) se sienten seguros caminando de noche y solos por su barrio. La sensación de seguridad disminuye en los estratos más bajos.
Robo de objetos personales con violencia, según estrato sociodemográfico

El porcentaje de habitantes que han sufrido un robo con violencia fueron: el $77,9 \%$ de habitantes de estrato sociodemográfico alto, el $89,1 \%$ de personas del sociodemográfico medio alto, el $85,4 \%$ de los habitantes que son estrato sociodemográfico medio y finalmente el $88,5 \%$ del estrato sociodemográfico medio bajo. Es decir, en estos cuatro estratos más de las cuatro quintas de los encuestados manifestaron haber sido víctimas de un robo con violencia.

Percepción del nivel de delincuencia en su distrito, según el nivel sociodemográfico

Entre los habitantes del estrato sociodemográfico alto, $8,7 \%$ consideran que la delincuencia ha disminuido, el $60.1 \%$ cree que se mantiene igual, mientras que un $31,2 \%$ considera que ha aumentado. En el estrato sociodemográfico medio alto consideran que la delincuencia ha disminuido el 7,8\%, el 36.4\% considera que se mantiene igual y el $55,8 \%$ cree que ha aumentado. El 5,2\% de las personas del estrato sociodemográfico medio cree que la delincuencia ha disminuido, el $18,8 \%$ siente que se mantiene igual y el $76,0 \%$ considera que ha aumentado. Por último, en el estrato medio bajo, el 6,7\% manifiesta que ha disminuido la delincuencia, $17,0 \%$ considera que se mantiene igual y el $76,3 \%$ cree que ha aumentado. Se puede apreciar que, con excepción del estrato alto, los encuestados consideran en su mayoría (más de la mitad) que la delincuencia ha aumentado, especialmente en los estratos más bajos.

\section{Confianza en el servicio policial, según el estrato sociodemográfico}

El $15,9 \%$ de los habitantes de estrato sociodemográfico alto indican que es poco confiable el servicio policial, el 42,3\% considera que el servicio policial es nada confiable y el $38,0 \%$ creen que es algo confiable. En el estrato sociodemográfico medio alto, el $41,9 \%$ de las personas juzgan que el servicio policial es poco confiable y el $27,9 \%$ consideran que el servicio policial es nada confiable. En el estrato sociodemográfico medio el 34,4\% manifiestan que el servicio policial es poco confiable y el $33,8 \%$, que es nada confiable. Finalmente, entre los encuestados del estrato medio bajo, el 35,4\% manifiestan que el servicio policial es poco confiable y el $41,7 \%$ consideran que es nada confiable. Como se ve, en los diferentes estratos, 
la mayoría de los entrevistados creen que el servicio policial es o poco confiable o nada confiable.

Percepción de la vigilancia en su distrito por la policía, según estrato sociodemográfico

El porcentaje de encuestados que consideran que en su distrito hay vigilancia por parte de la Policía Nacional es como sigue: $72,1 \%$ de los habitantes del estrato sociodemográfico alto, el $72,1 \%$ de los habitantes del nivel medio alto, el $57,0 \%$ de los correspondientes al estrato sociodemográfico medio y el 55,2\% de los entrevistados del nivel medio bajo. Como se aprecia, la percepción de que haya vigilancia policial en el distrito de residencia disminuye en los estratos más bajos.

Percepción de cuán seguro(a) se siente caminando en la calle solo(a), según estrato sociodemográfico

Entre los entrevistados del nivel sociodemográfico alto, el $0.5 \%$ se sienten muy seguros; el 44,2 indican que se sienten seguros, el 53,9\% manifiestan que se sienten inseguros y el 1,4\% señalan que se sienten muy inseguros caminando solos(as) en la calle. En el estrato sociodemográfico medio alto, el 18.6\% declaran sentirse seguros, el $76,7 \%$ manifiestan sentirse inseguros y el $4,7 \%$ indican que se sienten muy inseguros. El $6,7 \%$ de los entrevistados del estrato medio indicaron sentirse seguros, el $84,7 \%$ declararon sentirse inseguros y el $8,6 \%$ señalaron sentirse muy inseguros. Finalmente, el 9,5\% de los entrevistados del estrado sociodemográfico medio bajo declararon sentirse seguros, mientras que el 83,2\% indicaron sentirse inseguros y el 7,3\% señalaron que se sentían muy inseguros. En los tres últimos estratos mencionados, ningún entrevistado señaló sentirse muy seguros al caminar en la calle solos. En este indicador también se aprecia que la percepción de inseguridad aumenta en los estratos más bajos.

Talcomoseapreciaenlatabla 1, todos losindicadores de percepción de inseguridad están significativamente asociados con el estrato sociodemográfico. Como ya se mencionó, la percepción de inseguridad en general es mayor en los estratos sociodemográficos bajos. Además, se obtuvo que el nivel de relación entre los indicadores y el estrato sociodemográfico varía entre medio y bajo.

Percepción de cuán seguros(as) se sienten en casa, según el distrito de residencia

El 1,3\% de los entrevistados del distrito de Lima manifestaron sentirse muy seguros en su propia casa; el $53,2 \%$, seguros; el $42,6 \%$, inseguros y el $2,9 \%$, muy

Tabla 1. Prueba chi cuadrado y coeficiente de contingencia de la relación entre indicadores de percepción de inseguridad y estrato sociodemográfico.

\begin{tabular}{|c|c|c|}
\hline Variables & $\begin{array}{l}\text { Chi cuadrado } \\
(p \text { valores })\end{array}$ & Coeficiente de contingencia Phi de Pearson \\
\hline Cómo se siente en casa & $\begin{array}{c}<0,001 \\
\text { Existe relación }\end{array}$ & $\begin{array}{l}0,347 \\
\text { Nivel medio de asociación }\end{array}$ \\
\hline $\begin{array}{l}\text { Cómo se siente caminando de noche y } \\
\text { solo/a por su barrio }\end{array}$ & $\begin{array}{c}<0,001 \\
\text { Existe relación }\end{array}$ & $\begin{array}{l}0,443 \\
\text { Nivel medio de asociación }\end{array}$ \\
\hline $\begin{array}{l}\text { Robo de objetos personales con violen- } \\
\text { cia }\end{array}$ & $\begin{array}{c}0,002 \\
\text { Existe relación }\end{array}$ & $\begin{array}{c}0,130 \\
\text { Nivel bajo de asociación }\end{array}$ \\
\hline La delincuencia en su distrito & $\begin{array}{l}\quad<0,001 \\
\text { Existe relación }\end{array}$ & $\begin{array}{c}0,360 \\
\text { Nivel medio de asociación }\end{array}$ \\
\hline Confianza en el servicio policial & $\begin{array}{l}<0,001 \\
\text { Existe relación }\end{array}$ & $\begin{array}{c}0,231 \\
\text { Nivel bajo de asociación }\end{array}$ \\
\hline $\begin{array}{l}\text { Vigilancia en el distrito por parte de la } \\
\text { Policía Nacional }\end{array}$ & $\begin{array}{c}0,011 \\
\text { Existe relación }\end{array}$ & $\begin{array}{c}0,166 \\
\text { Nivel bajo de asociación }\end{array}$ \\
\hline $\begin{array}{l}\text { Cómo se siente caminando por la calle } \\
\text { solo/a }\end{array}$ & $\begin{array}{l}\quad<0,001 \\
\text { Existe relación }\end{array}$ & $\begin{array}{c}0,371 \\
\text { Nivel medio de asociación }\end{array}$ \\
\hline
\end{tabular}

Fuente: INEI, 2017 
inseguros. En el caso del distrito de San Isidro, 7,8\% de los encuestados informaron sentirse muy seguros en sus casas; $84,5 \%$, seguros; $6,8 \%$, inseguros y $0,9 \%$, muy inseguros. Se aprecia una gran diferencia entre ambos distritos en este indicador, notándose que en el caso de San Isidro, más de 9 entre 10 entrevistados afirmaron sentirse muy seguros o seguros, en tanto que estas categorías cubren solamente un poco más de la mitad de los encuestados del distrito de Lima.

Percepción de cómo se siente caminando de noche y solo(a) por su barrio, según el distrito de residencia El 0,3\% de los habitantes del distrito de Lima se sienten muy seguros caminando de noche solo(a) por su barrio, el 9,5\% seguros, el 70,4\% inseguros y el $19,8 \%$ muy inseguros. En el caso del distrito de San Isidro, informan el $1,5 \%$ de los entrevistados que se sienten muy seguros, el 60,7\%, seguros; el 35,0\%, inseguros y el 2,8\%, muy inseguros. Como se ve, la percepción de seguridad al caminar de noche y solo(a) por el barrio es mucho mayor en el distrito comercial y residencial de San Isidro.

Robo de objetos personales con violencia, según el distrito de residencia

El 87,6 \% de los entrevistados del distrito de Lima declararon haber sido víctimas de robo de objetos personales con violencia, en el caso del distrito de San Isidro esa cifra alcanzó el 77,7\% de los encuestados. En ambos distritos existe una alta incidencia de este tipo de delitos, pero esta incidencia es algo mayor en el distrito de Lima.

Percepción del nivel de delincuencia en su distrito, según el distrito de residencia

El 70,4\% de los habitantes del distrito de Lima consideran que la delincuencia en su distrito ha aumentado, el 24,6\% consideran que se mantiene igual y $5,0 \%$ consideran que ha disminuido. En el caso del distrito de San Isidro, el 26,7\% de los entrevistados creen que la delincuencia ha aumentado en su distrito; $63,6 \%$ percibe que la delincuencia en su distrito se mantiene igual y $9,7 \%$ cree que la delincuencia ha disminuido. Nuevamente, la mayor percepción de inseguridad corresponde al distrito de Lima.

\section{Confianza en el servicio policial según el distrito de residencia}

De los entrevistados del distrito de Lima manifiestan sólo el 2,1\% consideran que es muy confiable el servicio policial, mientras que el 33,9\% no tiene nada de confianza en dicho servicio. En el caso del distrito de San Isidro, el 3,9\% consideran que es muy confiable el servicio policial, pero el $15,5 \%$ de los entrevistados respondieron que no tienen nada de confianza con este servicio. Se aprecia que el nivel de confianza en el servicio que presta la policía es algo más elevado en el distrito de San Isidro.

Tabla 2. Prueba chi cuadrado y coeficiente de contingencia de la relación entre indicadores de percepción de inseguridad y distrito de residencia

\begin{tabular}{|c|c|c|}
\hline Variables & $\begin{array}{l}\text { Chi cuadrado } \\
\text { ( } p \text { valores })\end{array}$ & Coeficiente de contingencia Phi de Pearson \\
\hline Cómo se siente en casa & $\begin{array}{c}<0,001 \\
\text { Existe relación }\end{array}$ & $\begin{array}{c}0,374 \\
\text { Nivel medio de asociación }\end{array}$ \\
\hline $\begin{array}{l}\text { Cómo se siente caminando de noche y } \\
\text { solo/a por su barrio }\end{array}$ & $\begin{array}{l}<0,001 \\
\text { Existe relación }\end{array}$ & $\begin{array}{c}0,491 \\
\text { Nivel medio de asociación }\end{array}$ \\
\hline Robo de objetos personales con violencia & $\begin{array}{c}0,002 \\
\text { Existe relación }\end{array}$ & $\begin{array}{c}0,129 \\
\text { Nivel bajo de asociación }\end{array}$ \\
\hline La delincuencia en su distrito & $\begin{array}{l}<0,001 \\
\text { Existe relación }\end{array}$ & $\begin{array}{c}0,388 \\
\text { Nivel medio de asociación }\end{array}$ \\
\hline Confianza en el servicio policial & $\begin{array}{l}\quad<0,001 \\
\text { Existe relación }\end{array}$ & $\begin{array}{c}0,211 \\
\text { Nivel bajo de asociación }\end{array}$ \\
\hline $\begin{array}{l}\text { Vigilancia en el distrito por parte de la } \\
\text { Policía Nacional }\end{array}$ & $\begin{array}{c}0,096 \\
\text { No existe relación }\end{array}$ & - \\
\hline $\begin{array}{l}\text { Cómo se siente caminando por la calle } \\
\text { solo/a }\end{array}$ & $\begin{array}{l}<0,001 \\
\text { Existe relación }\end{array}$ & $\begin{array}{c}0,396 \\
\text { Nivel medio de asociación }\end{array}$ \\
\hline
\end{tabular}

Fuente: INEI, 2017 
Percepción de cuán seguro(a) se siente caminando en la calle solo(a), según distrito de residencia

En relación a este aspecto de percepción de seguridad, el $10,1 \%$ de los entrevistados del distrito de Lima declararon que se sentían seguros, $82,7 \%$, manifestaron que se sentían inseguros y 7,2\% indicaron que se sentían muy inseguros. En el caso de San Isidro, el $0,5 \%$ se sienten muy seguros; $47,0 \%$, seguros; $51,5 \%$, inseguros y $1,0 \%$, se muestran muy inseguros. Por tanto, es posible afirmar que existe un mayor porcentaje de personas provenientes del distrito de Lima en comparación a San Isidro que se sienten más inseguros.

Percepción de vigilancia en el distrito por parte de la Policía Nacional, según distrito de residencia

En el distrito de Lima, el $62,4 \%$ de los entrevistados saben que cuentan con un servicio policial en su distrito, un $36,0 \%$ dice no contar con el servicio policial y el restante $1,6 \%$ declara no tener conocimiento de ello. Asimismo, en el distrito de San Isidro, 70,9\% saben que cuentan con el servicio policial dentro de su distrito; $27,2 \%$ dicen no contar y $1,9 \%$ no saben al respecto.

En la tabla 2 se muestra el análisis de la relación entre los indicadores de percepción y el distrito de procedencia del entrevistado. Como puede verse, la mayoría de los indicadores de percepción están relacionados con el distrito, notándose que la mayor percepción de inseguridad se encuentra en el distrito de Lima. Este resultado es en gran medida consistente con el de la tabla 1, ya que, como se indicó, el distrito de Lima alberga una población que, en promedio, se ubica en un estrato sociodemográfico más bajo que el de San Isidro. Además, se obtuvo que el nivel de relación entre los indicadores y el distrito, oscila entre medio y bajo.

Percepción acerca de cuán seguros(as) se sienten en su casa, según sexo.

Entre los hombres, el 3,1\% se sienten muy seguros dentro de sus casas, $68,2 \%$ se sienten seguros, $27,3 \%$ se sienten inseguros y $1,4 \%$ declaran sentirse muy inseguros. En el caso de las mujeres, 4,8\% manifestaron sentirse muy seguras, $60,4 \%$ indicaron que se sentían seguras, 32,6\% reportaron sensación de inseguridad y $2,2 \%$ señalaron sentirse muy inseguras. Por tanto, los hombres reportaron con más frecuencia sensación de seguridad dentro de sus casas, aunque la diferencia entre sexos en este aspecto no es muy grande.

Percepción de cuán seguros(as) se sienten caminando de noche y solo(a) por su barrio, según sexo

En el grupo de hombres, el 0,7\% manifestaron sentirse muy seguros; $31,5 \%$, seguros; $55,6 \%$,

Tabla 3. Prueba chi cuadrado y coeficiente de contingencia de la relación entre indicadores de percepción de inseguridad y sexo

\begin{tabular}{lcc}
\hline Variables & $\begin{array}{c}\text { Chi cuadrado } \\
(\boldsymbol{p} \text { valores })\end{array}$ & Coeficiente de contingencia Phi de Pearson \\
\hline Cómo se siente en casa & 0,189 \\
No existe relación & - \\
Cómo se siente caminando de noche y & 0,197 & - \\
solo/a por su barrio & No existe relación & - \\
Robo de objetos personales con violencia & 0,918 & - \\
& No existe relación & - \\
La delincuencia en su distrito & 0,926 & - \\
& No existe relación & 0,109 \\
Confianza en el servicio policial & 0,814 & Nivel bajo de asociación \\
Vigilancia en el distrito por parte de la & No existe relación & - \\
Policía Nacional & 0,030 & Existe relación \\
Cómo se siente caminando por la calle & 0,136 & No existe relación \\
solo/a &
\end{tabular}

Fuente: INEI, 2017 
inseguros y $12,2 \%$, muy inseguros. En el caso de las mujeres, el $0,7 \%$ reportaron sentirse muy seguras; 23,8 , seguras; $60,1 \%$ inseguras y $15,4 \%$ muy inseguras. Como en el caso anterior, la percepción de inseguridad se da con más frecuencia entre las mujeres.

\section{Robo de objetos personales con violencia, según sexo}

El 83,9\% de los varones entrevistados reportaron que fueron víctimas de robo de objetos personales con violencia, en el caso de las mujeres, $84,2 \%$ informaron haber experimentado este tipo de incidente. La gran similitud entre estos porcentajes indica que la diferencia de sexo no constituye un factor determinante para ser víctima de robos con violencia.

Percepción del nivel de la delincuencia en su distrito, según sexo

El 6,3\% de los hombres entrevistados consideran que la delincuencia en su distrito ha disminuido, el $38,8 \%$ perciben que mantiene igual y el $54,9 \%$ creen que ha aumentado. Por otro lado, en cuanto a las mujeres, $7,1 \%$ reportan que la delincuencia en su distrito ha disminuido, el $37,9 \%$ consideran que se mantiene igual y el 55,0\% perciben que ha aumentado. En ambos sexos, y de modo casi idéntico, la percepción de aumento del nivel de delincuencia es relativamente alta (más de la mitad de entrevistados).

Confianza en el servicio policial, según sexo

Entre los hombres entrevistados, 2,4\% considera el servicio policial muy confiable, $30,8 \%$ lo percibe algo confiable, $37,8 \%$ lo considera poco confiable y el $29,0 \%$ lo evalúa nada confiable. En cuanto al grupo femenino, el 3,0\% percibe el servicio policial muy confiable, $30,9 \%$ consideran este servicio algo confiable, $40,3 \%$ lo creen poco confiable y $25,8 \%$ lo consideran nada confiable. En ambos sexos, la percepción de baja confiabilidad en el servicio policial es mayoritaria.

Percepción de la vigilancia en su distrito por la policía, según sexo

El $61,2 \%$ de los hombres y el $69,5 \%$ de las mujeres que conformaron la muestra consideran que sí hay vigilancia en el distrito de la policía nacional.

Percepción de cuán seguro(a) se siente caminando en la calle solo(a), según sexo
El 5,9\% de los entrevistados del grupo masculino manifestaron que se sienten muy seguros mientras caminan solos en la calle, 26,2\% indicaron que se sienten seguros y $67,9 \%$ se mostraron inseguros. En el caso de las mujeres, $0,3 \%$ dijeron sentirse muy seguras, $20,1 \%$ indican que se sienten seguras, $75,5 \%$ se sienten inseguras y, por último, $4,1 \%$ reportaron sentirse muy inseguras al momento de caminar sola por la calle. Por tanto, también en este indicador existe un nivel algo mayor de inseguridad en el caso de las mujeres.

Aunque, en general, hay una mayor tendencia a la sensación de inseguridad entre las mujeres, los datos de la tabla 3 indican que sólo en el indicador de percepción de vigilancia del distrito por la policía existe una diferencia significativa según el sexo del entrevistado.

\section{DISCUSIÓN}

Los resultados indican que las variables de estrato sociodemográfico y distrito de residencia están relacionadas con todas las variables de percepción de inseguridad ciudadana. En general, se percibe más inseguridad en los estratos sociodemográficos más bajos, y en el distrito de Lima, en comparación con San Isidro, considerando que el primero está habitado por ciudadanos que, en promedio, corresponden a un nivel sociodemográfico más bajo que el de San Isidro. Una gran mayoría de personas del estrato sociodemográfico alto se sienten seguros en sus casas y creen que su distrito está constantemente vigilado por la policía. En este estrato, además, más de la mitad considera que el nivel de delincuencia no ha aumentado, en contraste con los restantes estratos en los que más de la mitad de los entrevistados sí considera que la delincuencia ha aumentado. Asimismo, entre los ciudadanos del estrato medio bajo, cerca del $40 \%$ no se sienten seguros en su propia vivienda y sólo un poco más de la mitad considera que hay vigilancia policial en su distrito. En todos los estratos, la sensación de seguridad disminuye fuera de casa. La mayoría de los entrevistados, especialmente en los estratos más bajos, no tiene confianza en la labor policial. Más del 70\% informaron haber sido víctimas de robo con violencia. La comparación entre Lima y San Isidro en cada uno de los indicadores mencionados arroja resultados paralelos a los de la comparación entre los estratos sociodemográficos más altos y más bajos.

La variable sexo, se obtuvo que solo estuvo relacionada con la variable de percepción de la 
existencia de vigilancia en el distrito por parte de la Policía Nacional, mostrando que el sexo no es una variable determinante en la percepción de inseguridad en los habitantes, al menos entre los indicadores considerados. Este resultado es similar al obtenido en algunos estudios realizados en otros países, por ejemplo el de Medina (2003) en España, quien igualmente halló que el miedo al delito no estaba relacionado con el género, aunque dicho autor sí mencionó que tal resultado no es coherente con lo reportado en el resto de la literatura. Lo que se esperaría es que las personas de sexo femenino se sientan físicamente más vulnerables frente a la acción delictiva: sin embargo, también debe considerarse que en el caso del presente estudio nos encontremos frente a un temor generalizado al delito, producto del nivel de violencia que en los últimos años han alcanzado los actos delictivos en la ciudad de Lima

Las notorias diferencias en la percepción de la inseguridad en función del nivel sociodemográfico tienen paralelo en lo reportado generalmente en la literatura. Hernández (2016) resaltó que la relación entre la incidencia del delito de y la desigualdad económica es significativa y negativa. Es probable que en este aspecto tengan incidencia una serie de factores más bien objetivos, como el hecho de que los distritos en que habitan ciudadanos de un mayor nivel adquisitivo cuenten en general con mejores servicios de seguridad, en los cuales no sólo participa la policía, sino también el servicio municipal de seguridad (o serenazgo), así como servicios privados de seguridad; los cuales puede que tengan menor presencia en los distritos habitados por ciudadanos con menor poder adquisitivo. La encuesta de Lima Cómo Vamos (2018), por ejemplo, indica que la percepción de inseguridad en la ciudad es igual en todos los sectores socioeconómicos en Lima y Callao, pero se incrementa en los sectores más bajos, cuando es lo que se evalúa es la percepción de la inseguridad en el lugar concreto de residencia (barrio). Por otro lado, es un hallazgo reportado recurrentemente que las personas de los niveles socioeconómicos más bajos se sientan más inseguros, vulnerables o temerosos frente a la delincuencia (e. g. Pantazis, 2000, Cali Cómo Vamos, 2017).

En segundo lugar, otro elemento objetivo que debe considerarse consiste en la alta tasa de robos con violencia, en todos los estratos, de los que han sido víctimas los entrevistados. Estos dos hechos podrían ser determinantes en la percepción de inseguridad reportada. Sin embargo, como se ha hecho notar
(López, 2014), la victimización no explica por sí sola la percepción de inseguridad. Es decir, que incluso el hecho de no haber sido víctima no exime a alguien de sentirse inseguro. Existen muchos factores que pueden explicar la sensación de inseguridad independientemente del grado real de victimización. Consideremos, por ejemplo, el factor de desconfianza frente a la labor policial. Este último aspecto se ha señalado como un posible determinante del miedo al delito; es decir, que "no es solamente el fenómeno objetivo del delito en sí lo que está impulsando el miedo al delito, sino también el fracaso de la policía para obtener la confianza de los ciudadanos" (Dammert y Malone, 2006, p. 32). Este factor de desconfianza parece estar más o menos generalizado a nivel global. En el presente estudio, en todos los estratos existe un elevado nivel de desconfianza frente a la labor policial y, según lo que estamos señalando, este hecho puede contribuir fuertemente al incremento de la sensación de inseguridad en Lima. Los resultados nos indican, en suma, que los habitantes de la ciudad de Lima, representados por algunos informantes de los distritos de Cercado de Lima y de San Isidro, pueden estar considerándose como prácticamente indefensos y completamente expuestos a ser víctimas de los delincuentes.

\section{Correspondencia}

\author{
Alison Aguilar Inga \\ Correo electrónico: alison.aguilar@upch.pe
}

\section{Referencias}

Cali Cómo Vamos (2017). Encuesta de percepción ciudadana. Cali: Programa Cali Cómo Vamos. Recuperado de: https://www.calicomovamos.org.co/ encuesta-percepcion-ciudadana

Comité Distrital de Seguridad Ciudadana de San Isidro (2018). Plan Local de Seguridad Ciudadana. San Isidro, Lima, Perú: Municipalidad de San Isidro. Recuperado de http://msi.gob.pe/portal/seguridad/codisec/plan-localde-seguridad-ciudadana

Dammert, L. (2012). Fear and crime in Latin America, Redefying state-society relations. Nueva York: Routledge. Dammert, L. \& Malone, M. F. T. (2006). Does it take a village? Policing strategies and fear of crime in Latin America. Latin America Politics \& Society, 48, 27-51. García-Castro, J. D. \& Villalobos, M. (2016). ¿Vivimos en un lugar seguro? Percepción de criminalidad en la Zona de Occidente. Revista Pensamiento Actual, 16, 285-294.

Garland, D. (2005). La cultura del control. Crimen y orden social en la sociedad contemporánea. Barcelona: 
Gedisa.

Hale, C. (1996). Fear of crime: A review of literature. International Journal of Victimology, 4, 79-150.

Hernández, W. (2016). Teorías y evidencias del “dilema urbano” en el Perú: ¿Por qué crecimos económicamente con violencia? (2000-2012). Economía, 39, 145-185.

Instituto Nacional de Estadística e Informática (2017a). Estadística de seguridad ciudadana.

Lima: Instituto Nacional de Estadística e Informática. Recuperado de https:/www.inei.gob.pe/media/ MenuRecursivo/boletines/seguridad_ciudadana_ mar2017.pdf

Instituto Nacional de Estadística e Informática (2017b). Encuesta Nacional especializada sobre victimización. Lima: Instituto Nacional de Estadística e Informática. Recuperado de http:/iinei.inei.gob.pe/iinei/srienaho/ Descarga/FichaTecnica/608-Ficha.pdf

Jasso, C. (2013). Percepción de inseguridad en México. Revista Mexicana de Opinión Pública, 15, 13-29.

Kessler, G. (2009). El sentimiento de inseguridad. Sociología del temor al delito. Buenos Aires: Siglo XXI. Lima Cómo Vamos. (2018). Encuesta Lima Cómo Vamos 2018. IX Informe de percepción sobre calidad de vida en Lima y Callao. Lima: Lima Cómo Vamos. Recuperado de: http://www.limacomovamos.org/cm/wp-content/ uploads/2018/12/EncuestaLimaComoVamos2018.pdf
López, N. (2014). Inseguridad y percepción de inseguridad en Lima, Perú. Cuadernos de Investigación, 10, 3-44.

Medina, J. (2003). Inseguridad ciudadana, miedo al delito y policías en España. Revista Electrónica de Ciencia Penal y Criminología, 05(03):0-0.

Municipalidad Metropolitana de Lima (2017). Plan Local de Seguridad Ciudadana.

Lima: Municipalidad Metropolitana de Lima. Recuperado de http:/www.munlima.gob.pe/images/descargas/ codisec/180417-PLAN-LOCAL-SEGURIDADCIUDADANA-2017-02.pdf

Ortiz de Urbina, I., Pareja, M., Ponce, J. \& Sibina, D. (2006). Estudio preliminar:

convivencia cuidadana, seguridad pública y urbanismo. En: I. Ortiz de Urbina \& J. Ponce (cords.), Convivencia ciudadana, seguridad pública y urbanismo. Diez textos fundamentales del panorama internacional (pp. 11-68). Madrid: Fundación Democracia y Gobierno Local.

Pantazis, C. (2000). Fear of crime, vulnerability and poverty. British Journal of Criminology, 40, 414-436.

Rottenbacher, J. M., Amaya, L., Genna, K. \& Pulache, M. (2009). Percepción de inseguridad ciudadana y su relación con la ideología política en una muestra de habitantes de la ciudad de Lima. Revista Española de Investigación Criminológica, 7, 1-22. 